\title{
A spotlight on alirocumab in high cardiovascular risk patients with type 2 diabetes and mixed dyslipidemia: a review on the emerging data
}

This article was published in the following Dove Press journal: Diabetes, Metabolic Syndrome and Obesity: Targets and Therapy

\author{
Khyatiben Rana' \\ Jessica Reid' \\ Joshua N Rosenwasser ${ }^{2}$ \\ Todd Lewis ${ }^{3}$ \\ Mae Sheikh-Ali ${ }^{4}$ \\ Rushab R Choksi ${ }^{1}$ \\ Rebecca F Goldfaden' \\ 'East Coast Institute for Research, \\ Jacksonville, FL, USA; ${ }^{2}$ Lincoln Medical \\ and Mental Health Center, Bronx, NY, \\ USA; ${ }^{3}$ Baker-Gilmour Cardiovascular \\ Institute, Jacksonville, FL, USA; \\ ${ }^{4}$ Northeast Florida Endocrine and \\ Diabetes Associates, Jacksonville, \\ FL, USA
}

\begin{abstract}
Diabetes is a significant and independent risk factor for atherosclerotic cardiovascular disease (ASCVD), leading to morbidity and mortality among this population. The prevention of macrovascular complications, such as CVD, peripheral arterial disease, and cerebrovascular accident, in patients with diabetes is obtained through multifactorial risk reduction, including mixed dyslipidemia management and adequate glycemic control. For patients with diabetes, it is crucial to initiate adequate dyslipidemia therapy to achieve recommended low-density lipoprotein cholesterol (LDL-C) goal of $<70 \mathrm{mg} / \mathrm{dL}$ or target non-high-density lipoprotein goal of $<100 \mathrm{mg} / \mathrm{dL}$. Lipid-lowering therapies (LLTs), such as statins and ezetimibe, are the cornerstone for plasma LDL-C lowering; however, individuals with diabetes are often unable to achieve target lipid goals with these therapies alone and frequently require additional treatments. A new class of LLTs, proprotein convertase subtilisin-kexin type 9 (PCSK9) inhibitors, provides a novel approach to lowering lipids in persons with high $\mathrm{CV}$ risk, such as those with diabetes. The clinical data presented in this review indicate the potential benefits of alirocumab in patients with diabetes and its value as a treatment option in patients with diabetic dyslipidemia with no significant safety concerns.
\end{abstract} Keywords: praluent, alirocumab, PCSK9, PCSK9 inhibitor, cardiovascular disease, atherosclerosis, dyslipidemia

\section{Introduction}

Over the last few decades, there has been a drastic increase in the prevalence of type 2 diabetes (T2D) within the United States, with numbers reaching over 30 million people in 2015. ${ }^{1,2}$ This is of significant concern, as a two-to-four-fold increase in cardiovascular disease (CVD) is observed in individuals with diabetes and diabetes-associated macrovascular complications such as CVD, peripheral arterial disease (PAD), and cerebrovascular accident, have been and remain the primary cause of morbidity and mortality in the diabetic population. ${ }^{3,4}$ As a result, it has been well established that having a diagnosis of diabetes, alone, is an independent risk factor for numerous macrovascular complications. Additionally, when diabetes is accompanied with dyslipidemia, the co-existence of the two chronic conditions plays a substantial role in the accelerated development of CVD. ${ }^{5}$

CVD commences with the presence of an abnormal lipid profile that is characterized by decreased high-density lipoprotein cholesterol (HDL-C) and elevated triglycerides (TG) and low-density lipoprotein cholesterol (LDL-C). In patients with T2D, although there is an increase in TG levels, very low-density lipoprotein
Correspondence: Rebecca F Goldfaden East Coast Institute for Research, Jacksonville, FL, USA

Tel + I 904854 I354

$\mathrm{Fax}+19048541355$

Email Rebecca.Goldfaden@ecirmed.com 
(VLDL), and intermediate-density lipoprotein (IDL) and decrease in HDL-C levels, the LDL-C levels are similar in patients without diabetes. Nevertheless, the levels of oxidized LDL-C that may be associated with pro-atherogenic properties are increased. ${ }^{6}$ Additionally, in patients with diabetes who have an abnormal lipid profile and when combined with persistent hyperglycemia, elevated blood pressure, and an increase in oxidative stress, long-term endothelial damage can occur. ${ }^{3,7}$ LDL-C commonly accumulates within the damaged endothelium of blood vessels and, overtime, contributes to the formation of atherosclerotic plaque. This plaque can either partially or completely occlude the blood vessels leading to PAD and/or ischemic heart disease. Most concerning is the vulnerable plaque, in which unstable areas can rupture and form a thrombus resulting in a myocardial infarction (MI) or an ischemic stroke. ${ }^{8}$ In individuals with diabetes, good glycemic control can help mitigate dyslipidemia and decrease the acceleration of atherosclerotic plaque formation. ${ }^{9}$ However, a certain degree of dyslipidemia persists despite the attempt to normalize blood glucose levels. ${ }^{9}$ Therefore, in patients with diabetes, the need for aggressive therapy to treat dyslipidemia is mandated to reduce the risk of cardiovascular (CV) morbidity and mortality. ${ }^{9}$ In this review, we will discuss the limitations of currently available lipid-lowering therapies (LLTs) that lead to the utilization of PCSK9 inhibitors, evaluate the clinical trials with alirocumab (Praluent), and evaluate the clinical efficacy and safety of utilizing alirocumab for the treatment of diabetes-mixed dyslipidemia.

\section{LDL-C and its metabolism}

LDL-C has been the primary target of LLTs for numerous years due to the substantial data demonstrating a direct correlation to a reduction in CVD and mortality. Therefore, the metabolic pathway of LDL-C is crucial to understanding the therapeutic approaches in reducing atherosclerotic cardiovascular disease (ASCVD). Four major classes of lipoproteins exist and include VLDL, HDL, LDL, and chylomicrons. ${ }^{10}$

The endogenous pathway for lipid metabolism commences when the liver produces VLDL, which contains many TGs and apolipoprotein B-100 (apoB) as a core structural protein. VLDL undergoes metabolism by lipoprotein lipase within the bloodstream, releasing TGs, resulting in the formation of a more cholesterol-enriched IDL. After further metabolism of the IDL and release of additional TGs, the LDL particle is produced and the buried apoB is exposed. ${ }^{11}$ The final LDL product is cholesterol-enriched and contains the apoB on its surface which is the ligand required for binding to LDL receptors (LDL-R) located on the hepatocyte cell membranes. ${ }^{11}$

LDL-Rs are key regulators of plasma LDL-C levels and are responsible for approximately $70 \%$ of plasma LDL-C clearance by the liver. $^{12,13}$ The active form of the sterol regulatory element-binding protein (SREBP) transcription factors is responsible for mediating the expression of LDLRs when cholesterol levels are low within the liver. SREBPs accomplish this by stimulating the transcription of LDL-R and 3-hydroxy-3-methylglutaryl coenzyme A (HMG-CoA) reductase, a key enzyme in cholesterol biosynthesis that is involved in the rate-limiting step of converting HMG-CoA to mevalonic acid. ${ }^{13}$ LDL-Rs carry cholesterol into the hepatocyte by binding to the apoB ligand on the circulating LDL-C, producing the LDL-LDL-R complex which mediates endocytosis. After the internalization of the endocytic vesicle, the LDL-R dissociates from the LDL-LDL-R complex and recycles back to cell surface to be utilized again, leaving the LDL to be hydrolyzed within the cytosol. ${ }^{11}$ Each LDL-R can be recycled up to 150 times before they are endocytosed and degraded. Conversely, when cholesterol levels are high within the hepatocyte, SREBP remains in its inactive form and prevents the transcription of LDL-Rs. $^{14}$

In individuals with dyslipidemia, plasma LDL-C remains abnormally elevated and dysregulation of LDLRs impairs the normal homeostasis of LDL metabolism. ${ }^{1}$ Under such conditions, LDL-C builds up and deposits on arterial walls. ${ }^{1}$ This process is accelerated when LDL-C is oxidized due to the presence of increased free radicals and insulin resistance in individuals with diabetes. Oxidized LDL-C induces an inflammatory response and attracts macrophages that transform them into foam cells. These physiological changes in the LDL particles result in a higher number of small, dense LDL that significantly increases the risk of atherosclerotic plaque and ASCVD. ${ }^{15}$

\section{Pharmacological approaches for the management of diabetic dyslipidemia}

\section{Statins}

In individuals with T2D, mixed dyslipidemia is often underdiagnosed as plasma LDL-C levels usually remain within the normal range. Therefore, a better indicator of $\mathrm{CV}$ risk in this population is non-HDL-C since it accounts for all atherogenic lipoproteins, including LDL, IDL, 
VLDL, remnants of VLDL, remnants of chylomicrons, and lipoprotein(a) $(\mathrm{Lp}(\mathrm{a})){ }^{15}$ Statins are a class of LLT that increase the clearance of all atherogenic lipoproteins and decrease plasma cholesterol concentration. The American Diabetes Association (ADA) guidelines recommend the use of moderate-intensity statins as first-line agents for individuals 40-75 years of age with diabetes and without ASCVD whereas high-intensity statins are recommended for persons of all ages with diabetes and ASCVD. ${ }^{16}$ This recommendation is very similar to the 2018 American College of Cardiology/American Heart Association (ACC/AHA) guidelines for blood cholesterol, where the initiation of statins in persons 40-75 years of age with diabetes is strongly recommended, irrespective of their ASCVD risk. ${ }^{17}$

The reduction of intracellular cholesterol, more particularly LDL-C, by statins is achieved by inhibiting HMG-CoA reductase. This leads to an upregulation of LDL-R on the surface of hepatocytes and results in an increase in the removal of LDL-C from the periphery. ${ }^{18}$ Numerous clinical trials studying the effects of statins have shown them to be highly efficacious in lowering plasma LDL-C levels, with an approximate 30-50\% reduction. ${ }^{12}$ Nevertheless, despite the great lipid-lowering capabilities of statins, persons with T2D frequently fail to achieve target plasma LDL-C levels of $<70 \mathrm{mg} /$ $\mathrm{dL}$ and often require additional LLTs. ${ }^{12}$ Furthermore, the LDL-C lowering potential of statins may be limited in individuals who are unable to tolerate them due to possible side effects, such a myalgias, or in individuals with extremely high LDL-C levels such as those with familial hypercholesterolemia (FH). Fortunately, the emergence of monoclonal antibodies that inhibit PCSK9 has shown substantial positive data in reducing plasma LDL-C while demonstrating a favorable side effect profile in persons whose LDL-C cannot be managed by statins or other LLTs, such as ezetimibe.

\section{PCSK9 inhibitors}

\section{PCSK9 mechanism of action}

PCSK9 is a crucial protein for cholesterol homeostasis. The wild-type PCSK9 gene located on chromosome 1 was first described in 2003 and is primarily expressed in the liver. $^{19,20}$ Within the liver, PCSK9 binds to the first EGF-like repeat A located on the LDL-Rs and shuttles the LDL-Rs intracellularly into the lysosomes for degradation. This process results in fewer LDL-Rs on hepatocyte cell membrane leading to reduced LDL-C uptake and increased plasma LDL-C levels (Figure 1). ${ }^{12}$

The relationship of PCSK9 to CVD was first demonstrated through numerous genetic studies that identified the association between mutations in the PCSK9 gene with plasma LDL-C levels. Genetic variants of gain-of-function mutations in the PCSK9 gene were shown to be linked with autosomal dominant diseases, homozygous FH (HoFH) and heterozygous FH (HeFH). ${ }^{1,19}$ In individuals with these conditions, the increase in PCSK9 leads to a decrease in the production of LDL-Rs. This results in an insufficient uptake and breakdown of LDL within the hepatocytes resulting in an increased level of circulating LDL. ${ }^{1}$ Conversely, patients with a dysfunctional PCSK9 gene have a significantly lower plasma LDL-C levels with a noticeable decrease in $\mathrm{CV}$ events whereas, patients with a complete loss of function of the PCSK9 gene have plasma LDL-C levels that may be lower than $20 \mathrm{mg} / \mathrm{dL} .^{8}$ Thus, it is established that inhibition of PCSK9 can play a significant role in reducing plasma LDL-C concentrations and the risk of CVD.

\section{PCSK9 inhibitors mechanism of action and their effect in patients with T2D}

The novel discovery of fully humanized monoclonal antibodies against PCSK9, also known as PCSK9 inhibitors, has revolutionized the treatment of hypercholesterolemia. There are two commercially available subcutaneous PCSK9 inhibitors in the US, alirocumab (Praluent) and evolocumab (Repatha). Both medications are monoclonal antibodies (mAbs) that neutralize PCSK9 activity by binding to the catalytic domain of PCSK9 and blocking its interaction with LDL-Rs. This action results in decreased degradation of the LDL-Rs, enabling more LDL-Rs to recycle back to the hepatocyte membrane to increase the plasma LDL-C clearance (Figure 1). ${ }^{20}$

The discovery of these agents has been recent with alirocumab (Praluent) gaining US Food and Drug Administration (FDA) approval in July 2015 and evolocumab (Repatha) obtaining FDA approval in August 2015. Nevertheless, due to their profound ability to decrease LDL-C, they have been included as a treatment option in the 2018 American College of Cardiology/American Heart Association (ACC/AHA) guidelines for the management of blood cholesterol. ${ }^{17}$ In this, they are indicated for the primary prevention of $\mathrm{CV}$ events in persons with multiple risk factors and have LDL-C $\geq 100 \mathrm{mg} / \mathrm{dL}$ despite receiving a combination of a maximal statin therapy and ezetimibe. Similarly, when LLTs, such as maximally tolerated statins and 

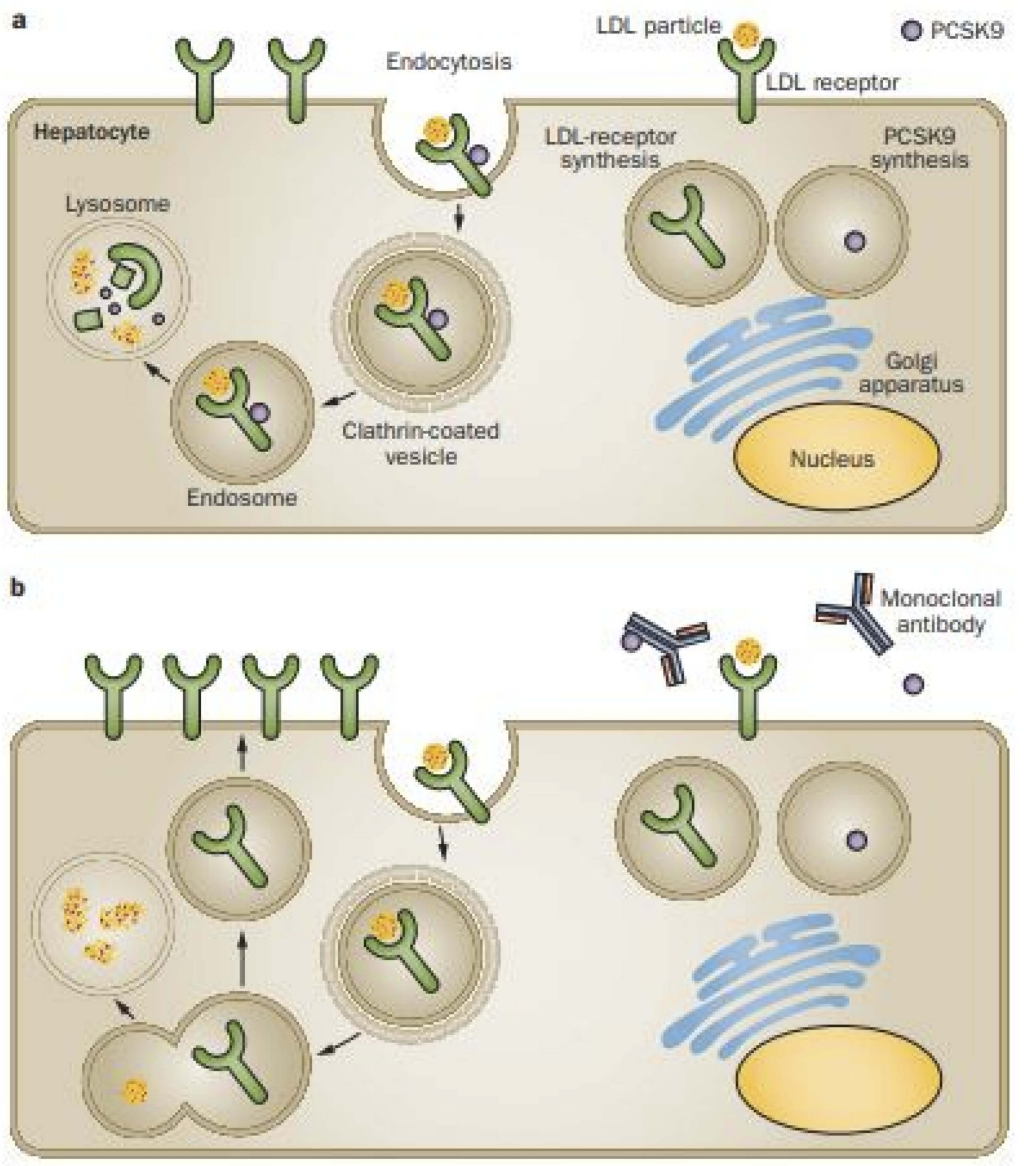

Figure I PCSK9 inhibitors mechanism of action. (A) PCSK9 is primarily secreted in the liver and acts as a key mediator in LDL-C metabolism. PCSK9 inhibits LDL-R recycling by targeting LDL-R and promoting lysosomal degradation. Overall, this process results in a reduction of LDL-Rs and a reduction in plasma LDL-C clearance. (B) Monoclonal antibodies, such as alirocumab, bind to PSCK9 and inhibit it from binding to LDL-Rs. This enables more LDL-Rs to recycle back to cell surface resulting in increased LDL-C clearance. Reprinted with permission from Springer Nature: Springer Nature, Nature Reviews Cardiology, Lipid lowering with PCSK9 inhibitors, Dadu RT, Ballantyne CM, Copyright (20I4). ${ }^{23}$

Abbreviations: PCSK, proprotein convertase subtilisin-kexin type 9; LDL-C, low-density lipoprotein cholesterol; LDL-R, LDL receptors.

ezetimibe are unsuccessful in allowing individuals with diabetes who have ASCVD to achieve the ADA goals of plasma LDL-C $<70 \mathrm{mg} / \mathrm{dL}$, PSCK9 inhibitors are recommended as an add-on therapy. ${ }^{16}$ Such commendations from these two guidelines reflect from the positive results of numerous clinical trials that have demonstrated that when PCSK9 inhibitors are added to background LLTs in high CV risk patients, including those with diabetes, they are more likely to achieve a significant reductions in various lipid parameters and aid in reducing the risk of premature ASCVD in this population. ${ }^{21}$

\section{Alirocumab}

Alirocumab (Praluent) is currently indicated as a treatment option in patients who are on a maximum-tolerated statins with HeHF or with ASCVD and require additional LLT. ${ }^{18}$ The efficacy of alirocumab was studied in high CV risk patients, including those with diabetes, and showed a significant reduction in plasma LDL-C and apoB levels compared to controls. In addition, alirocumab demonstrated significant LDL-C lowering by up to $70 \%$ when used with statin therapy, indicating both independent and additive effects with statins in patients with diabetes. ${ }^{22}$ Finally, an expected reduction in alirocumab out-of-pocket cost for Medicare patients to approximately $\$ 25$ to $\$ 150$ USD per month, may make it a more affordable therapeutic option for patients who are unable to achieve desired LDL-C goals with statin and/or ezetimibe.

\section{Phase I - alirocumab clinical trials}

Prior to describing the alirocumab data as it relates to diabetes and dyslipidemia, the Phase I and Phase II results must be reviewed to provide ample background knowledge. Three 
Phase I clinical trials were conducted with alirocumab in individuals without diabetes and no known ASCVD. These studies included two single-dose studies in healthy volunteers and one multi-dose study in individuals with either HeFH $(n=21)$ or non-FH $(n=41) .{ }^{24}$ In the single-dose studies, individuals had serum LDL-C levels $>100 \mathrm{mg} / \mathrm{dL}$ and were prohibited from using non-study LLTs. These individuals received alirocumab either intravenously $(\mathrm{IV} ; \mathrm{n}=40)$ with weight-based dosing $(0.3,1.0,3.0,6.0$, or $12.0 \mathrm{mg} / \mathrm{kg})$ or subcutaneously $(\mathrm{SC})(\mathrm{n}=32)$ at various set doses $(50,100$, or $150 \mathrm{mg}){ }^{25,26}$ The multi-dose study comprises three cohorts of individuals: 1) $\mathrm{HeFH}$ with LDL-C $>100 \mathrm{mg} / \mathrm{dL}$ on atorvastatin, 2) non-FH with LDL-C $>100 \mathrm{mg} / \mathrm{dL}$ on atorvastatin, and 3) non-FH with LDL-C $>130 \mathrm{mg} / \mathrm{dL}$ being treated with only modified diet. ${ }^{24,27}$

Patients in the single-dose study receiving IV alirocumab achieved a percent change in LDL-C from baseline versus placebo of $-28.1 \%,-42.4 \%,-57.4 \%,-56.5 \%$, and $-65.4 \%$ for the $0.3 \mathrm{mg} / \mathrm{kg}, 1.0,3.0,6.0$, and 12.0 $\mathrm{mg} / \mathrm{kg}$ doses, respectively $\left(P<0.001\right.$ for all). ${ }^{24}$ A difference in percent change from baseline of LDL-C versus placebo for patients in the single-dose subcutaneous study was $-32.5 \%,-39.9 \%,-38.5 \%,-45.7 \%$ for the 50,100 , 150 , and $250 \mathrm{mg}$ doses, respectively $\left(P<0.001\right.$ for all). ${ }^{24}$

In the multi-dose study, looking at both the FH and non-FH patients combined who were on atorvastatin, at day 57 posttreatment with alirocumab 50,100, and $150 \mathrm{mg}$, treated individuals achieved a percent change of LDL-C from baseline versus placebo of $-39.2 \%,-53.7 \%$, and $-61.0 \%$, respectively $(P<0.001$ for all). Similarly, individuals treated with diet and exercise achieved a percent change of LDL-C from baseline versus placebo of $-57.0 \%$ when treated with alirocumab $150 \mathrm{mg}$ ( $P=0.0023$ ). When examining the other lipid parameters, the study demonstrated a percent change of total cholesterol (TC) from baseline versus placebo of $-24.6 \%,-33.2 \%,-36.4 \%$ $(P<0.0001$ for all) with alirocumab 50,100, and $150 \mathrm{mg}$ in the atorvastatin treated individuals and $-43.3 \%(P=0.0025)$ with alirocumab $150 \mathrm{mg}$ in the individuals treated with diet and exercise. Furthermore, there was a significant percent change of apoB from baseline versus placebo of $-31.5 \%,-42.0 \%$, and $-46.4 \%$ with alirocumab 50,100 , and $150 \mathrm{mg}$ groups, respectively $(P<0.0001$ for all $){ }^{24}$

Overall, the Phase I trials demonstrated the extent of LDL-C reduction with alirocumab was dose-dependent with higher doses leading to greater LDL-C reductions. Furthermore, in the multi-dose trial, alirocumab's efficacy in the various dose groups corresponded to a reduction of free PCSK9 measured in the plasma. In addition, the effects of alirocumab were observed in individuals only 2 weeks after the first dose in all three cohorts, indicating a quicker onset of action compared to statin therapies. Most importantly, alirocumab resulted in great LDL-C lowering despite background treatment with atorvastatin and regardless of a diagnosis of FH. This data confirmed that alirocumab has an additive effect on LDL-C lowering when used with statin therapy as the same LDL-C reductions were shown when alirocumab was delivered alone or in addition to atorvastatin. ${ }^{24}$

\section{Phase II - alirocumab studies}

Phase II studies further evaluated the addition of alirocumab to statin therapy. Patients included in the Phase II studies were required to undergo a 6-week washout or stabilization period on a statin with or without ezetimibe prior to randomization and continued on this regimen throughout the study. ${ }^{28}$ Each trial was to evaluate the effect of alirocumab on LDL-C compared with placebo in patients with LDL-C $\geq 100 \mathrm{mg} / \mathrm{dL}$ in addition to ongoing stable atorvastatin therapy. These studies included patients with FH, but excluded patients with T1D and T2D requiring insulin and/or $\mathrm{HbA} 1 \mathrm{c}$ values $\geq 8.5 \%$. $^{28,29}$

One Phase II study had five treatment groups at randomization with varying doses of alirocumab, including doses higher than that studied in the Phase I trials. The five treatment groups included alirocumab $150 \mathrm{mg}$ SC every 4 weeks (Q4W) (n=15), $200 \mathrm{mg} \mathrm{SC} \mathrm{Q4W} \mathrm{(n=16),} \mathrm{or} 300 \mathrm{mg}$ SC Q4W ( $=15), 150 \mathrm{mg}$ SC every 2 weeks (Q2W) $(n=16)$, and placebo Q2W $(n=15)$. Patients in the alirocumab treatment groups had a least-squares (LS) mean percent reduction of $-28.9 \%,-31.5 \%,-42.5 \%$ and $-67.9 \%$ in LDL-C from baseline to week 12 , respectively, versus $-10.65 \%$ in the placebo group $(P<0.0001$ for all). The number of patients who achieved LDL-C concentrations $<100 \mathrm{mg} / \mathrm{dL}$ at week 12 was greatest in the alirocumab $150 \mathrm{mg} \mathrm{SC} \mathrm{Q2W}$ $(\mathrm{n}=15 ; 94 \%)$ compared to alirocumab $150 \mathrm{mg}$ SC Q4W $(\mathrm{n}=4 ; 27 \%), 200 \mathrm{mg}$ SC Q4W ( $=3 ; 19 \%), 300 \mathrm{mg} \mathrm{SC}$ Q4W $(n=10 ; 67 \%)$, and placebo $(n=2 ; 13 \%)$. The alirocumab $150 \mathrm{mg}$ SC Q2W dose also showed significant improvements in non-HDL-C, TC and apoB and HDL-C. ${ }^{28}$

Another Phase II trial examined alirocumab co-administered with atorvastatin (10 $\mathrm{mg}$ to $80 \mathrm{mg})$ without other LLTs. ${ }^{30}$ Patients, including those with T2D (15\% of total population), were randomized to atorvastatin $80 \mathrm{mg}$ PO daily $(\mathrm{n}=31)$, atorvastatin $10 \mathrm{mg}$ PO daily plus 
alirocumab $150 \mathrm{mg} \mathrm{Q2W} \mathrm{(n=31),} \mathrm{or} \mathrm{atorvastatin} 80 \mathrm{mg}$ PO daily plus alirocumab $150 \mathrm{mg} \mathrm{Q} 2 \mathrm{~W}(\mathrm{n}=30) .{ }^{29}$ The combination of atorvastatin $80 \mathrm{mg}$ PO daily plus alirocumab 150 mg Q2W showed significant reductions in LDL-C compared to atorvastatin $80 \mathrm{mg}$ PO daily alone with an LS mean reduction of $-73.2 \%$ compared to $-17.3 \%$, respectively $(P<0.001)$. In addition, the combination of atorvastatin $10 \mathrm{mg}$ PO daily plus alirocumab $150 \mathrm{mg}$ Q2W resulted in an LS mean reduction of $-66.2 \%$. Furthermore, a target LDL-C goal of $<100 \mathrm{mg} / \mathrm{dL}$ was achieved by all patients treated with alirocumab $150 \mathrm{mg} \mathrm{SC} \mathrm{Q} 2 \mathrm{~W}$ compared to $52 \%$ of those treated with atorvastatin $80 \mathrm{mg}$ PO daily alone, and at least $90 \%$ of those treated with alirocumab $150 \mathrm{mg} \mathrm{SC}$ Q2W achieved LDL-C levels $<70 \mathrm{mg} / \mathrm{dL}$ versus $17 \%$ with atorvastatin $80 \mathrm{mg}$ PO daily alone. ${ }^{29}$

A final Phase II study included patients on stable atorvastatin therapy $(10,20$ or $40 \mathrm{mg}$ PO daily) for $\geq 6$ weeks and prohibited the use of other LLTs. This study randomized patients to alirocumab $50 \mathrm{mg} \mathrm{SC}$ Q2W $(\mathrm{n}=30)$, alirocumab $100 \mathrm{mg}$ SC Q2W (n=31), alirocumab $150 \mathrm{mg} \mathrm{Q2W} \mathrm{(n=31),}$ placebo SC Q2W (n=31) or alirocumab $200 \mathrm{mg}$ SC Q4W $(n=30)$ or alirocumab $300 \mathrm{mg}$ SC Q4W ( $n=30)$ while continuing their same atorvastatin dose. Out of the total study population, approximately $12 \%$ of the patients had T2D. LDL-C reductions were consistent across atorvastatin doses and showed a dose-response pattern for alirocumab Q2W and Q4W dosing. The study results demonstrated an LS mean percent change in LDL-C from baseline to week 12 for each group of $39.6 \%$ for alirocumab $50 \mathrm{mg} \mathrm{SC} \mathrm{Q2W,} \mathrm{64.2 \%} \mathrm{for}$ alirocumab $100 \mathrm{mg} \mathrm{SC} \mathrm{Q2W,} \mathrm{72.42 \%} \mathrm{for} \mathrm{alirocumab} 150 \mathrm{mg}$ SC Q2W, 43.2\% for alirocumab $200 \mathrm{mg}$ SC Q4W and 47.7\% for alirocumab $300 \mathrm{mg} \mathrm{SC} \mathrm{Q4W,} \mathrm{and} 5.1$ with placebo. In addition, alirocumab substantially reduced non-HDL-C and apoB. $^{31}$

All data from the Phase II trials were pooled, totaling 352 patients with hypercholesterolemia on background statins with or without ezetimibe. Throughout the various Phase II trials, the study patients received alirocumab doses ranging from 50 to $300 \mathrm{mg} \mathrm{SC}$ Q2W or Q4W. ${ }^{32}$ These studies further validated alirocumab's dose-related and frequency-related (e.g., Q2W vs Q4W) efficacy on LDL-C reduction, which were clearly independent of background atorvastatin dose. ${ }^{31}$

\section{Phase III - alirocumab studies}

Alirocumab was then studied in a large Phase III program, the ODYSSEY program, which consisted of numerous clinical trials studying alirocumab $75 \mathrm{mg}$ SC Q2W with a potential for a blinded titration to $150 \mathrm{mg} \mathrm{SC} \mathrm{Q2W} \mathrm{at}$ week 12 if the patient's LDL-C was $\geq 70 \mathrm{mg} / \mathrm{dL}$ at week 8 of treatment (hereby referred as "alirocumab $75 \mathrm{mg} / 150$ mg SC Q2W") in several patient populations. Two Phase III trials (ODYSSEY FH I and ODYSSEY FH II) conducted specifically in the HeFH population enrolled a total of 735 subjects. Patients were included if they had a diagnosis of $\mathrm{HeFH}$ and their LDL-C level was $>100 \mathrm{mg} /$ $\mathrm{dL}$ (if treated for primary prevention) or $>70 \mathrm{mg} / \mathrm{dL}$ (if treated for secondary prevention) despite maximally tolerated statin therapy (i.e., atorvastatin $40-80 \mathrm{mg}$, rosuvastatin $20-40 \mathrm{mg}$, or simvastatin $80 \mathrm{mg}$, or max tolerated dose of one of these statins with documented reason) with or without other LLTs. Patients were randomized in a 2 to 1 fashion to either alirocumab $75 \mathrm{mg} / 150 \mathrm{mg} \mathrm{SC}$ Q2W or placebo for 78 weeks. At week 24, a placebo-corrected LDL-C reduction of $-57.9 \%$ and $-51.4 \%$ was demonstrated in the ODYSSEY FH I trial and ODYSSEY FH II trial, respectively ( $P<0.0001$ for both), which was maintained through week 78 . Furthermore, with $9 \%$ of the total study population having a diagnosis of T2D at baseline, the trial results demonstrated that diabetes did not have an impact on the efficacy of alirocumab. Further, when looking at the potential effects of alirocumab on glycemic control, there were no numerical differences in the development or worsening of diabetes in both ODYSSEY FHI and FH II populations. This was demonstrated by a mean change in HbAlc from baseline to week 78 of 0.03 compared to 0.02 for alirocumab vs placebo, respectively, in ODYSSEY FHI and 0.10 versus 0.03 in ODYSSEY FH II. Lastly, the percentage of patients reaching a target LDL-C of $<70 \mathrm{mg} / \mathrm{dL}$ at week 24 was $59.8 \%$ (vs $0.8 \%$ ) and $68.2 \%$ (vs $1.2 \%$ ) in the alirocumab versus placebo group $(P<0.0001))^{33}$

ODYSSEY ESCAPE was another HeFH trial that enrolled $\mathrm{HeFH}$ patients undergoing weekly or Q2W lipoprotein apheresis to remove apoB-containing lipoproteins from the blood. The 18 -week trial randomized 62 patients with $\mathrm{HeFH}$ in a 2 to 1 fashion to either alirocumab $150 \mathrm{mg}$ SC Q2W $(n=41)$ or placebo $(n=21)$. In the trial, the patient's lipoprotein apheresis rate was adjusted according to their response to treatment and pre-apheresis LDL-C value. Therefore, when the LDL-C level was $\geq 30 \%$ lower than the baseline (pre-apheresis) LDL-C value, apheresis was not performed. Alirocumab showed a statistically significant reduction in the number of apheresis treatments, with a $75 \%$ reduction in apheresis treatments (from weeks 7 to 18$)$ compared to those on placebo $(P<0.0001)$. 
Furthermore, during the 12-week treatment period, $63.4 \%$ of alirocumab patients did not require any apheresis treatments and $92.7 \%$ of alirocumab patients only required half of their usual apheresis treatments compared to the placebo group. This study showed that alirocumab could be used in conjunction with lipoprotein apheresis to reduce LDL-C and the frequency of lipoprotein apheresis required in patients with $\mathrm{HeFH}^{34}$

The ODYSSEY HIGH FH trial was a 24-week trial that compared alirocumab $150 \mathrm{mg}$ SC Q2W $(n=72)$ to placebo (35) in a 2 to 1 fashion amongst $\mathrm{HeFH}$ patients with an LDL-C $\geq 160 \mathrm{mg} / \mathrm{dL}$ on maximally tolerated statin therapy (defined as atorvastatin $40-80 \mathrm{mg}$, rosuvastatin $20-40 \mathrm{mg}$, or simvastatin $80 \mathrm{mg}$, or max tolerated dose of one of these statins with documented reason) with or without other LLTs. The results of the trial demonstrated an LS mean percentage change in LDL-C from baseline to week 24 of $-45.7 \%$ for alirocumab and $-6.6 \%$ for placebo, with a between group difference of $-39.1 \%(P<0.0001)$. This change corresponded to an LS mean absolute reduction in LDL-C of $-90.8 \mathrm{mg} / \mathrm{dL}$ for alirocumab compared to $-15.5 \mathrm{mg} / \mathrm{dL}$ for placebo $(P<0.0001) .{ }^{35}$ In this trial, approximately $14 \%$ of the total population had a diagnosis of T2D at baseline and the study outcomes did not report a difference in the worsening or development of diabetes between the groups (alirocumab: $1.4 \%[n=1]$; placebo: $2.9 \%[n=1])$.

The ODYSSEY LONG TERM trial included 2341 patients with LDL-C levels $\geq 70 \mathrm{mg} / \mathrm{dL}$ who either had $\mathrm{HeFH}$, established CHD, or a CHD risk equivalent. Prior to enrollment in the trial, all patients were on high-dose statin therapy or maximally tolerated statin therapy with or without other LLTs for at least 4 weeks. Treatment groups were assigned 2 to 1 for alirocumab $150 \mathrm{mg}$ SC Q2W $(n=1553)$ or placebo $(n=788)$ for 78 weeks. A difference in mean percentage change in LDL-C between alirocumab and placebo was $-61.9 \%(P<0.001)$ from baseline to week 24 , corresponding to $-61.0 \%$ in the alirocumab group versus $0.8 \%$ in the placebo group. This reduction was consistent over the trials' 78-week duration of therapy. Target LDL-C levels of $<70 \mathrm{mg} / \mathrm{dL}$ were achieved by $79.3 \%$ of the patients with alirocumab compared to $8.0 \%$ of those on placebo at week $24(P<0.001){ }^{22}$

The ODYSSEY Open-Label extension (OLE) study $(n=986)$ was a one-year extension trial of four ODYSSEY Phase III trials (FHI, FHII, LONG TERM, and HIGH HF) that assessed the long-term safety, efficacy, immunogenicity, and adherence to alirocumab in patients with HeFH when added to background LLTs. Six hundred and fifty-five patients that received alirocumab (one patient from FHI did not receive the treatment) and 330 patients that received placebo accounted for the 985 patients comprising the OLE study. The start of the OLE was synonymous with the end of the treatment period for the FHI, FHII and HIGH FH studies and patients in the FHI, FHII and HIGH FH studies were started on alirocumab $75 \mathrm{mg}$ SC Q2W. ${ }^{36}$ For patients who completed the LONG TERM study, there was an 8-week wash-out period at which time the LDL-C results were unblinded to physicians and titrations of alirocumab from $75 \mathrm{mg} \mathrm{SC} \mathrm{Q} 2 \mathrm{~W}$ to $150 \mathrm{mg} \mathrm{SC} \mathrm{Q} 2 \mathrm{~W}$ could occur starting at week $12 .{ }^{36} \mathrm{At}$ week 8 when LDL-C values were unblinded, a $44.2 \%$ reduction in LDL-C $(84.9 \mathrm{mg} / \mathrm{dL})$ was seen compared to the baseline LDL-C levels $(151.9 \mathrm{mg} / \mathrm{dL})$ in the parent studies. $^{36}$

In the ODYSSEY OLE trial, all patients received alirocumab $75 \mathrm{mg}$ or $150 \mathrm{mg}$ SC Q2W. Most patients (56.7\%) who started on alirocumab $75 \mathrm{mg} \mathrm{SC}$ Q2W in the ODYSSEY OLE trial continued that dose throughout the trial and $86.6 \%$ of patients from the ODYSSEY HIGH FH who began the parent trial on alirocumab $150 \mathrm{mg}$ SC Q2W remained on that dose throughout the ODYSSEY OLE study. The trial showed that patients' adherence to alirocumab treatment was high $(98.3 \%)$ with $99.6 \%$ of patients being at least $80 \%$ adherent and only $5.6 \%$ prematurely discontinuing treatment. ${ }^{37}$

Within the ODYSSEY OLE trial, $12 \%$ of the total population had a diagnosis of either T1D or T2D. Fortytwo patients in ODYSSEY OLE trial reported treatmentemergent adverse events (TEAEs) of diabetes or diabetes complications, 14 of which had diabetes at the OLE baseline and 28 did not. However, when looking only at the patients without diabetes $(n=867)$ at baseline of the ODYSSEY OLE trial, a similar incidence of diabetic mellitus or diabetic complications TEAEs were observed between patients treated with alirocumab or placebo in the parent studies $\left(3.4 \%\right.$ versus $2.9 \%$, respectively). ${ }^{36}$

The ODYSSEY COMBO I trial was a 52-week trial that studied 316 patients with a high ASCVD risk who either had an LDL-C $\geq 70 \mathrm{mg} / \mathrm{dL}$ with established CVD or LDL-C $\geq 100 \mathrm{mg} / \mathrm{dL}$ with coronary heart disease (CHD) risk equivalents (e.g., diabetes with other risk factors or chronic kidney disease [CKD]). Patients were randomized 2 to 1 to alirocumab $75 \mathrm{mg} / 150 \mathrm{mg} \mathrm{SC} \mathrm{Q2W}(\mathrm{n}=209)$ or placebo $(n=107)$ as an add-on to maximally tolerated statin therapy (defined as atorvastatin 40-80 $\mathrm{mg}$, rosuvastatin 
20-40 mg, or simvastatin $80 \mathrm{mg}$, or max tolerated dose of one of these statins with documented reason) with or without other LLTs. In the intention-to-treat population, the estimated mean percent change in LDL-C from baseline to week 24 was $-48.2 \%$ compared to $-2.3 \%$ in the alirocumab and placebo arms, respectively $(P<0.0001)$, which was sustained over the 52-week duration. At baseline, diabetes was represented in approximately $45 \%$ and $39.3 \%$ of the patients in the alirocumab and placebo arms, respectively, and alirocumab efficacy was not impacted by a diagnosis of diabetes at randomization. Furthermore, when looking at the total lipid profile, which is important for individuals with diabetes, alirocumab had positive effects on apoB (-35.8), non-HDL-C $(-37.5)$, TC (-25.0), and HDL-C (7.3) when examining the estimated percent change difference compared to placebo $(P<0.0001$ for all). Finally, a larger amount of patients were able to achieve an LDL-C goal of LDL-C $<70 \mathrm{mg} / \mathrm{dL}$ at week 24 with $75 \%$ in the alirocumab group and $9.0 \%$ in the placebo group $(P<0.0001){ }^{38}$

The ODYSSEY COMBO II trial was a 104-week trial studying alirocumab $(n=479)$ versus ezetimibe $(n=241)$ in patients with documented ASCVD (defined as CHD, PAD, or ischemic stroke) with an LDL-C $\geq 70 \mathrm{mg} / \mathrm{dL}$ and in patients without documented ASCVD but with other CV risk factors (e.g., patients with diabetes and $\geq 2$ risk factors) with an LDL-C $\geq 100 \mathrm{mg} / \mathrm{dL}$. All patients had to be maintained on maximally tolerated statin therapy, which was defined as atorvastatin 40-80 mg, rosuvastatin 20-40 $\mathrm{mg}$, or simvastatin $80 \mathrm{mg}$, or a max tolerated dose of one of these statins was allowed with a documented reason for intolerance to a higher dose. ${ }^{39}$ Patients enrolled in the ODYSSEY COMBO II trial were randomized in a 2 to 1 fashion to either alirocumab $75 \mathrm{mg} / 150 \mathrm{mg}$ SC Q2W or ezetimibe $10 \mathrm{mg}$ PO once daily. After 24 weeks of treatment, the mean percent reduction in LDL-C from baseline to week 24 was $-50.6 \%$ in the alirocumab group compared to $-20.7 \%$ in the ezetimibe group, demonstrating a between group difference of $-29.8 \% \quad(P<0.0001)$. This difference in the reduction of LDL-C between treatment groups remained consistent through follow-up at 52 weeks. $^{39}$

Within the ODYSSEY COMBO II trial $(n=720)$, approximately $31 \%(n=225)$ of the patients had an investigator-reported diagnosis of diabetes at baseline. Although the results of the trial demonstrated no difference in LDL-C reduction between those with or without diabetes, a sub-analysis was conducted to further investigate this potential concern. Baseline characteristics of the individuals with diabetes in the ODYSSEY COMBO II sub-analysis were a mean HbAlc of $6.8 \%$, duration of diabetes of 9.5 years, and approximately 82 $85 \%$ were receiving at least one anti-hyperglycemic agent prior to study entry. Of those receiving an anti-hyperglycemic agent, $22.3 \%$ and $16.9 \%$ were receiving insulin therapy at baseline in the alirocumab and ezetimibe groups, respectively. ${ }^{40}$

The ODYSSEY COMBO II sub-analysis demonstrated an LS mean percent change in LDL-C from baseline to week 24 of $-49.1 \%$ and $-51.2 \%$ for alirocumab-treated patients with and without diabetes $(P=0.8052)$. Furthermore, the mean percent difference in LDL-C with alirocumab versus ezetimibe at week 24 was $-30.7 \%$ in those with diabetes and $-29.5 \%$ in those without diabetes $(P=0.8025)$. Both non-statistically significant results indicate that alirocumab's efficacy did not differ between those with or without diabetes. Additionally, the LDL-C reductions were maintained over 104 weeks in both patient populations (with or without diabetes). ${ }^{40}$

In addition to alirocumab's benefits on LDL-C for patients with diabetes, the ODYSSEY COMBO II trial also found alirocumab to have favorable effects on the overall atherogenic lipid profile compared to ezetimibe, which is crucial in the treatment of diabetic dyslipidemia. When examining patients with and without diabetes, the trial showed similar differences between alirocumab and ezetimibe for non-HDL-C ( $-25.5 \%$ vs $-21.7 \%)$, Apo B $(-24.2 \%$ vs $-21.6 \%), \mathrm{Lp}(\mathrm{a})(-23.9 \%$ vs $-20.8 \%)$, and HDL-C $(7.1 \%$ and $8.5 \%)$ confirming equal efficacy in those with diabetes and those without diabetes. ${ }^{40}$ Lastly, the sub-study revealed that treatment with alirocumab resulted in a larger number of patients achieving a target LDL-C goal of $<70 \mathrm{mg} / \mathrm{dL}$ in both patients with diabetes $(77.9 \%)$ and without diabetes $(77.3 \%)$ compared to those treated with ezetimibe $(50.1 \%$ and $45.1 \%){ }^{40}$

Two trials within the ODYSSEY program, ODYSSEY DM-INSULIN and ODYSSEY DM-DYSLIPIDEMIA, were conducted specifically targeting a patient population with diabetes due to the historical concerns of statins being related to an increased risk of diabetes and the unknown nature of how PCSK9 affects glucose homeostasis. The ODYSSEY DM-INSULIN trial was a 24-week trial that assessed insulin-treated T2D patients $(n=441)$ and insulintreated Type 1 Diabetes (T1D) patients $(n=76)$ who had an LDL-C $\geq 70 \mathrm{mg} / \mathrm{dL}$ despite maximally tolerated statins with or without other LLTs. Furthermore, these patients 
had either a history of ASCVD (40.1\%) or at least one additional risk factor for CVD (59.9\%), in addition to having diabetes. Patients were randomized 2 to 1 to receive either alirocumab $75 \mathrm{mg} / 150 \mathrm{mg}$ SC Q2W $(n=345)$ or placebo $(n=172)$ as an add-on to their current LLTs and anti-hyperglycemic agents. Overall, only $22.6 \%$ of the total population in the alirocumab arm received a dose titration from $75 \mathrm{mg}$ to $150 \mathrm{mg}$, of which $20.5 \%$ of the patients had T2D and $36.7 \%$ of the patients had T1D. Although a larger number of T1D patients required an alirocumab dose titration, this number was proportional to the baseline LDL-C levels, which were $126.4 \mathrm{mg} / \mathrm{dL}$ for T1D compared to $110.8 \mathrm{mg} / \mathrm{dL}$ for T2D. This difference in baseline LDL-C levels was a likely cause for the difference in required dose titrations since six ODYSSEY trials identified that the baseline LDL-C levels have the greatest impact on the need for a dose titration. ${ }^{41}$

In general, when looking at the total ODYSSEY DMINSULIN patient population (both T1D and T2D), the LS mean percentage change in LDL-C from baseline to week 24 was $-50.1 \%$ for alirocumab and $-1.3 \%$ for placebo, demonstrating a between group difference of $-48.8 \%(P<$ 0.0001). When examining the T2D and T1D patients separately, similar results were demonstrated, indicating that the type of diabetes did not alter the efficacy of alirocumab. Within the T2D population, the LS mean percentage change in LDL-C from baseline to week 24 was $-48.2 \%$ for alirocumab and $+0.8 \%$ for placebo, revealing a between group difference of $-49.0 \%$ $(P<0.0001)$. Similarly, when analyzing the T1D patients alone, the LS mean percentage change in LDL-C from baseline to week 24 was $-51.8 \%$ for alirocumab and $-3.9 \%$ for placebo, displaying a difference between groups of $-47.8 \%(P<0.0001) .{ }^{41}$ When dividing the patients into further sub-groups within the T2D population and T1D population separately, the study showed that moderate $\mathrm{CKD}$, age, duration of diabetes, baseline $\mathrm{HbAlc}$, and history of ASCVD did not affect the efficacy of alirocumab. ${ }^{41}$

It is known that other lipid values are important in individuals with diabetes and alirocumab was found to have positive effects on various lipid parameters in the ODYSSEY DM-INSULIN trial. In T2D patients, alirocumab versus placebo resulted in significant reductions from baseline to week 24 for non-HDL-C $(-38.7 \%)$, apoB $(-36.7 \%)$, TC $(-27.6 \%)$ and $\mathrm{Lp}(\mathrm{a})(-18.4 \% ; P<0.0001$ for all), as well as a significant increase in HDL-C $(4.4 \% ; P<0.01)$. Similarly, in the T1D patients, alirocumab compared to placebo resulted in significant reductions from baseline to week 24 for non-HDL-C $(-42.7 \%)$, apoB $(-39.0 \%)$, TC $\left(-29.2 \% ; P<0.0001\right.$ for all). ${ }^{41}$

The ODYSSEY DM-INSULIN trial also evaluated specific diabetes-related endpoints, including $\mathrm{HbAlc}$, fasting plasma glucose (FPG), insulin total daily dose (TDD), and the number of anti-hyperglycemic agents utilized. Within the T2D patient population studied, the mean absolute change in $\mathrm{HbA} 1 \mathrm{c}$ from baseline to week 24 was $0.2 \%$ in the alirocumab arm and $0.1 \%$ in the placebo group and the mean absolute change in FPG from baseline to week 24 was $9.5 \mathrm{mg} / \mathrm{dL}$ and $10.0 \mathrm{mg} / \mathrm{dL}$, respectively. In the T1D population, the mean absolute change in $\mathrm{HbA} 1 \mathrm{c}$ from baseline to week 24 was $0.0 \%$ and $-0.2 \%$ and the mean absolute change in FPG from baseline to week 24 was $9.5 \mathrm{mg} / \mathrm{dL}$ and $14.6 \mathrm{mg} / \mathrm{dL}$ in the alirocumab and placebo groups, respectively. These changes in HbA1c and FPG are insignificant and reveal that alirocumab does not impact glycemic control. Furthermore, the insulin TDD and number of anti-hyperglycemic agents remained stable and did not change from baseline to week 24 indicating that alirocumab did not have an impact on the management of diabetes. ${ }^{41}$ Therefore, the ODYSSEY DM-INSULIN data demonstrates that patients with diabetes can achieve a similar degree of LDL-C reduction compared to patients without diabetes without any negative effects on their diabetes control. $^{41}$

The ODYSSEY DM-DYSLIPIDEMIA trial was a 24-week trial that randomized 413 T2D (HbA1c <9\%) patients with mixed dyslipidemia (non-HDL $\geq 100 \mathrm{mg} / \mathrm{dL}$ and TG between 150 and $500 \mathrm{mg} / \mathrm{dL}$ ) who were not controlled despite maximally tolerated statin doses (statin-intolerant patients allowed). Patients enrolled needed to either have a history of ASCVD or at least one additional risk factor for CVD in addition to having diabetes. ${ }^{15}$ As previously mentioned in this review, studying mixed dyslipidemia in the diabetic population is vital as LDL-C can often be controlled in this population despite having elevated levels of non-HDL-C, which encompasses all atherogenic lipoproteins.

The ODYSSEY DM-DYSLIPIDEMIA trial examined the effects of alirocumab versus usual care (UC), defined as maximally tolerated statin therapy, ezetimibe, fenofibrate, omega-3-fatty acids, or nicotinic acid. Patients were randomized 2 to 1 to receive either open-label alirocumab 75/150 mg SC Q2W ( $\mathrm{n}=276)$ or UC $(\mathrm{n}=137)$. UC for this trial was up to the choice of the investigator and was selected prior to randomization. ${ }^{15}$ The trial showed a statistically significant 
reduction in non-HDL-C with the LS mean percentage change from baseline to week 24 of $-37.3 \%$ and $-4.7 \%$ for alirocumab and UC, respectively, corresponding to a between group difference of $-32.5 \%$ vs UC $(P<0.0001)$. Both arms showed a decrease in TG levels with $-13.0 \%$ for alirocumab patients and $-8.8 \%$ for patients in the UC group ( $P=0.2191)$. Compared to UC, alirocumab also reduced apoB (-32.3\%), Lp(a) (-27.4\%), TC (-24.6\%) and LDL$\mathrm{C}(-43.0 \%)(P<0.0001$ for all $) .{ }^{15}$

The ODYSSEY DM-DYSLIPIDEMIA trial also evaluated specific diabetes-related endpoints, including $\mathrm{HbAlc}$ and FPG. The mean absolute change in $\mathrm{HbA1c}$ from baseline to week 24 was $0.24 \%$ in the alirocumab arm and $0.19 \%$ in the UC arm ( $P=0.48$ vs UC). In addition, the mean absolute change in FPG from baseline to week 24 was $5.70 \mathrm{mg} / \mathrm{dL}$ and $-0.10 \mathrm{mg} / \mathrm{dL}$ in the alirocumab arm and UC arm, respectively ( $P=0.012$ vs UC). Furthermore, the number of anti-hyperglycemic agents per patient remained stable throughout the duration of the study with no changes from baseline to week $24{ }^{15}$

The largest trial of the ODYSSEY program was the ODYSSEY OUTCOMES trial that enrolled 18,924 patients and evaluated CV outcomes after an acute coronary syndrome (ACS), defined as MI or unstable angina (UA), 1-12 months prior to randomization. It is known that patients with a previous ACS event, such as those included in this trial, are at high risk for recurrent ischemic $\mathrm{CV}$ events. Therefore, the primary measure for the ODYSSEY OUTCOMES study was time to first occurrence of Major Adverse Cardiac Event (MACE), which was defined as the first occurrence of CHD death, acute MI, hospitalization for UA or ischemic stroke in patients recently ( $<52$ weeks) hospitalized for ACS and on high-intensity statin therapy. ${ }^{42}$ Included patients had LDL-C of $\geq 70 \mathrm{mg} / \mathrm{dL}$, nonHDL of $\geq 100 \mathrm{mg} / \mathrm{dL}$ or apoB of $\geq 80 \mathrm{mg} / \mathrm{dL}$. All patients were on background treatment with atorvastatin $40-80 \mathrm{mg}$ PO daily or rosuvastatin $20-40 \mathrm{mg}$ PO daily ( $88.8 \%$ for both), or the maximum-tolerated dose of one of these two statins for at least 2 weeks. All patients were then randomized in a 1 to 1 fashion to either alirocumab $75 \mathrm{mg} \mathrm{SC} \mathrm{Q2W} \mathrm{(} \mathrm{n}=9462)$ titrated under blind conditions to a target LDL-C of $25-50 \mathrm{mg} / \mathrm{dL}$ or to placebo $(\mathrm{n}=9462){ }^{43}$

The results of the ODYSSEY OUTCOMES trial showed that the mean LDL-C levels greatly reduced from baseline $(92 \mathrm{mg} / \mathrm{dL})$, at 4 months $(-62.7 \%), 12$ months $(-61.0 \%)$, and 48 months $(-54.7 \%)$. Furthermore, the LDL-C reductions were sustained over the course of the trial and only 49 patients would need to be treated for 4 years to prevent the occurrence of one primary end-point event. $^{43}$

A subgroup analysis of ODYSSEY OUTCOMES was completed on patients with diabetes. ${ }^{44}$ At baseline, 5444 patients had history of either T1D or T2D and 8246 patients were defined as having prediabetes. The analysis showed across all patients with normoglycemia, diabetes or prediabetes, they all equally had greater reductions in LDL-C, non-HDL-C and TG levels compared with placebo and the overall absolute risk reduction (ARR) was $1.6 \%$ with a HR of $0.85(\mathrm{CI}=0.78-0.93)$. When examining patients with diabetes, prediabetes, and no diabetes separately, individuals with diabetes demonstrated an ARR of $2.3 \%$ and $\mathrm{HR} 0.84 \mathrm{CI}=(0.74-0.97)$, people with prediabetes had an ARR of $1.2 \%$ and HR 0.86 (CI=0.74-1.00), and patient with no diabetes showed an ARR of $1.2 \%$ and HR 0.85 ( $\mathrm{CI}=0.70-1.03)$. These results reveal that patients with diabetes had greater ARR compared to those without diabetes when dosed with alirocumab to target LDL-C levels of $25-50 \mathrm{mg} / \mathrm{dL}$ but had similar relative risk reductions. Lastly and most importantly, compared to placebo, there was no evidence of increased FPG, HbAlc, or newonset diabetes with alirocumab. ${ }^{44}$

\section{Pooled analysis of Phases II and III alirocumab studies}

A pooled analysis of 10 Phase III ODYSSEY trials $(n=4974)$ was conducted to further examine the effects of alirocumab $75 \mathrm{mg}$ or $150 \mathrm{mg}$ SC Q2W on diabetes compared to placebo and ezetimibe. Across all trials, the prevalence of diabetes (30.7\%), prediabetes (39.6\%), and normoglycemic $(29.7 \%)$ patients at baseline was comparable between treatment groups (ezetimibe-controlled or placebo-controlled). Overall, the pooled analysis found there was no increase in the incidence of diabetes in the alirocumab group compared with placebo $(\mathrm{HR}=0.64$; $\mathrm{CI}=0.36-1.14)$ or compared with ezetimibe $(\mathrm{HR}=0.55$; $\mathrm{CI}=0.22-1.41)$. In addition, the pooled analysis found there was no increase in the incidence of transition to prediabetes or diabetes in the alirocumab group compared with placebo $(\mathrm{HR}=1.20 ; \mathrm{CI}=0.96-1.49)$ or compared with ezetimibe $(\mathrm{HR}=0.88 ; \mathrm{CI}=0.59-1.32)$. Furthermore, $\mathrm{HbA} 1 \mathrm{c}$ changes seen from baseline were similar amongst alirocumab-treated patients and placebo $(0.04 \%$ and $0.05 \%$, respectively), as well as FPG changes $(0.36 \mathrm{mg} / \mathrm{dL}$ for both). ${ }^{45}$ This pooled analysis of 10 trials determined that there is no evidence that alirocumab affects the incidence 
of new-onset diabetes over a follow-up period of 6-18 months despite very significant LDL-C lowering of 40 $60 \%$ achieved. $^{45}$

Another pooled analysis of 14 Phase II and Phase III ODYSSEY trials $(n=5234)$ focused on the safety of alirocumab according to diabetes status. ${ }^{46}$ Approximately, $30.3 \%$ of the total population consisted of patients with diabetes, of which 1554 had T2D, 28 had T1D, and 2 had an unspecified form of diabetes. At baseline, the mean $\mathrm{HbA} 1 \mathrm{c}$ in all patients with diabetes was $6.9 \%$ for both the alirocumab and control groups compared to $5.7 \%$ for those without diabetes. In addition, baseline FPG levels in the alirocumab and control groups were $135 \mathrm{mg} / \mathrm{dL}$ in those with diabetes and $99 \mathrm{mg} / \mathrm{dL}$ in those without diabetes. Overall, $23.5 \%$ of those with diabetes were on insulin therapy prior to randomization. ${ }^{46}$ The results of this pooled analysis indicated that the safety of alirocumab was similar in patients with diabetes and without diabetes and further details of the safety are described within the adverse even section of this review. ${ }^{46}$

\section{Adverse events}

The adverse events (AEs) associated with alirocumab are highly published, therefore, for the purpose of this review a brief overview will be provided. ${ }^{33,35,38}$ Across all Phases I, II, and III clinical trials, alirocumab demonstrated a great safety profile with minimal treatment-related AEs. ${ }^{33,35,37,38}$ Potential general allergic reactions were reported from $6.9 \%$ to $12.5 \%$ in alirocumab-treated patients versus $5.2-12.1 \%$ in the placebo groups for the FHI, FHII, HIGH FH, ODYSSEY LONGTERM, ODYSSEY OLE, and COMBO I studies. ${ }^{22,33,35,36,38}$ Injection site reactions were identified across several trials, but these were classified as mild in intensity (a few moderate intensity), transient, and very few led to study drug discontinuation and ranged from $2.5 \%$ to $12.4 \%$ for alirocumab-treated patients versus $0.8-11 \%$ in the placebo groups over the FHI, FHII, HIGH FH, ODYSSEY ESCAPE, ODYSSEY LONGTERM, ODYSSEY OLE, COMBO I and COMBO II studies. ${ }^{22,33,34,35,36,38,39}$ Furthermore, when looking specifically at individuals with or without diabetes, patients with diabetes had a lower rate of injection site reactions than those without diabetes. This difference was likely related to the fact that patients with diabetes are more accustomed to receiving injectable therapies or performing needle sticks for selfmonitoring of blood glucose and reported less injection site reactions. ${ }^{33,35,38,40,41,46}$
An early concern for PCSK9 inhibitors was their ability to greatly reduce LDL-C levels to $<25 \mathrm{mg} / \mathrm{dL}$, which was initially thought to potentially cause cancer, hemorrhagic stroke, depression, and other AEs. The protocol for the ODYSSEY OUTCOMES trial specified if LDL-C was $<25 \mathrm{mg} / \mathrm{dL}$ on any 2 consecutive measurements with alirocumab $150 \mathrm{mg}$, the dose would be reduced to $75 \mathrm{mg}$ and LDL-C values that were $<15$ $\mathrm{mg} / \mathrm{dL}$ on 2 consecutive measurements with alirocumab $75 \mathrm{mg}$ would be discontinued and placebo injections would be substituted. ${ }^{42}$ However, after numerous longterm clinical trials, it was established that despite LDL$C$ reductions to $<25 \mathrm{mg} / \mathrm{dL}$, no safety concerns with alirocumab use were identified as a result. ${ }^{33,38}$ Another AE reported in clinical trials was treatment-emergent anti-drug antibodies (with alirocumab). Nevertheless, these anti-drug antibodies were not deemed to be clinically significant as alirocumab's efficacy was not negatively affected by their presence. ${ }^{33,35,38}$ Given that alirocumab was studied in a high CV risk population, it was important that the clinical trials with alirocumab proved it to have no impact on common comorbidities, such as blood pressure or diabetes. ${ }^{33,38,40,41}$ Finally and most importantly for this review, when comparing patients with or without diabetes, there was no significant difference in the overall safety profile of alirocumab and the AEs remained low across all groups, prediabetes, diabetes, or normoglycemic patients. ${ }^{45}$

\section{Conclusion}

The considerable amount of data presented in this review and summarized in Table 1 establishes that alirocumab should remain a valuable treatment option in patients with diabetic dyslipidemia. The ODYSSEY program, which showed superiority with respect to placebo in reducing MACE, such as MI, ischemic stroke, and all-cause mortality in patients with LDL $>100 \mathrm{mg} / \mathrm{dL}$ validates that individuals with diabetes will obtain equivalent lipid-lowering effects as those without diabetes, devoid of negative effects on glycemic control. Furthermore, no significant safety concerns are present in clinical trials or meta-analysis relating to the use of alirocumab in individuals with diabetes and thus, its utilization can greatly benefit these patients and enlighten the management of dyslipidemia in patients with diabetes. 


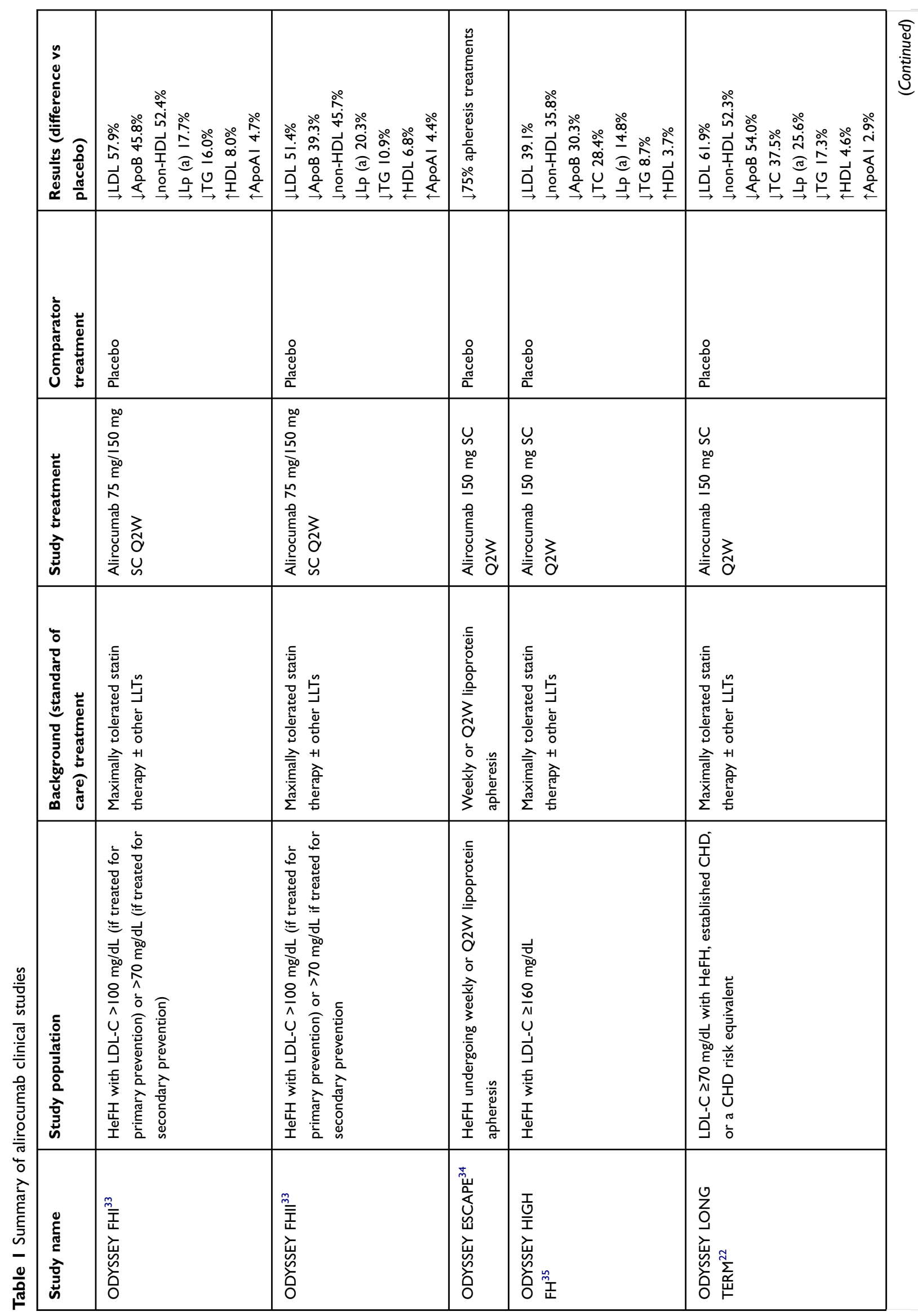




\begin{tabular}{|c|c|c|c|c|c|}
\hline 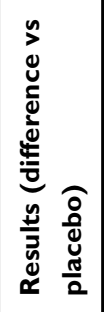 & 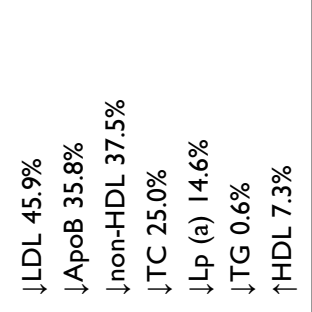 & 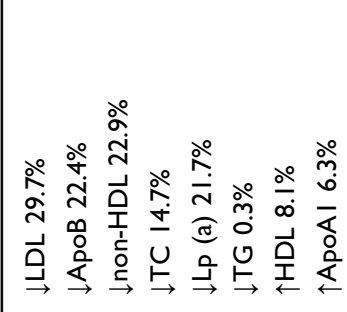 & 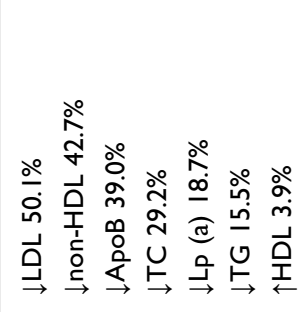 & 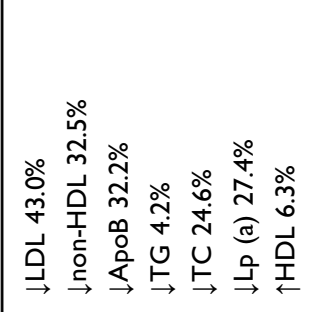 & 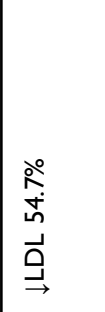 \\
\hline 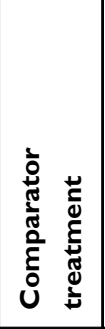 & $\begin{array}{l}\frac{0}{8} \\
\frac{\mathbb{g}}{0} \\
\end{array}$ & 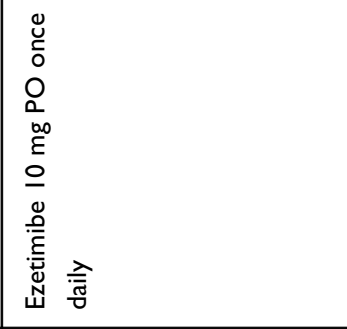 & $\begin{array}{l}\stackrel{\circ}{\frac{8}{0}} \\
\frac{\mathbb{\Xi}}{0}\end{array}$ & 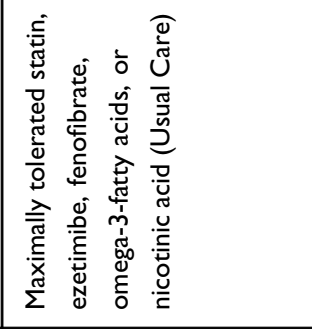 & $\begin{array}{l}\circ \\
\frac{8}{\mathbb{4}} \\
\frac{\pi}{a}\end{array}$ \\
\hline 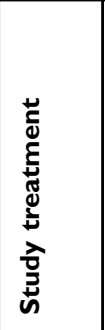 & 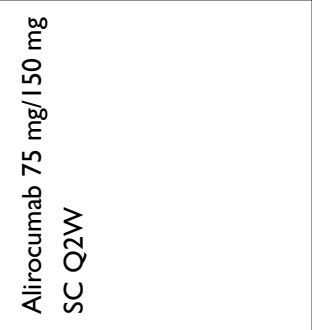 & 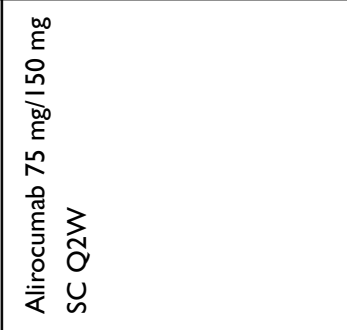 & 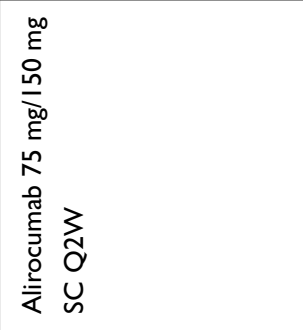 & 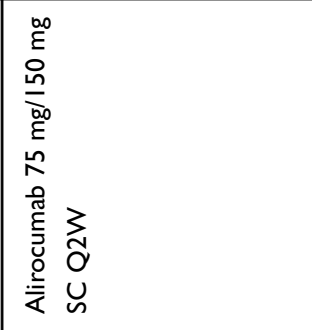 & 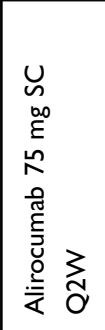 \\
\hline 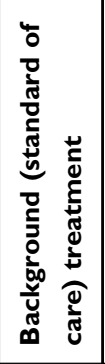 & 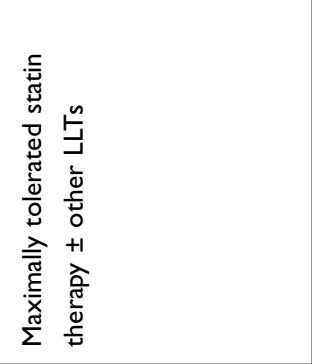 & 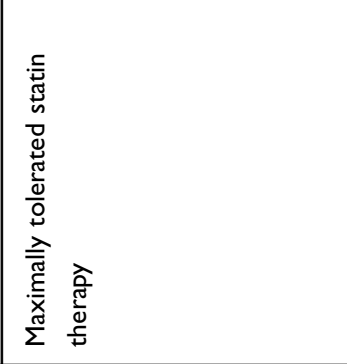 & 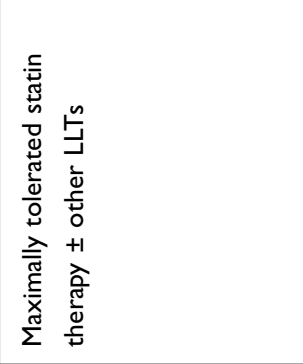 & 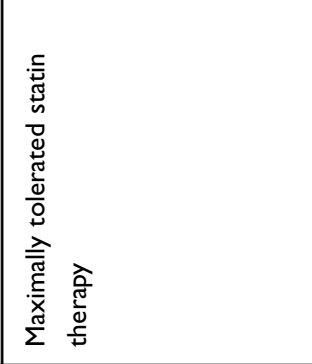 & 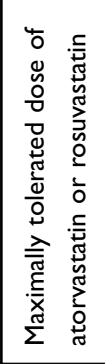 \\
\hline 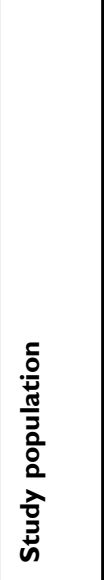 & 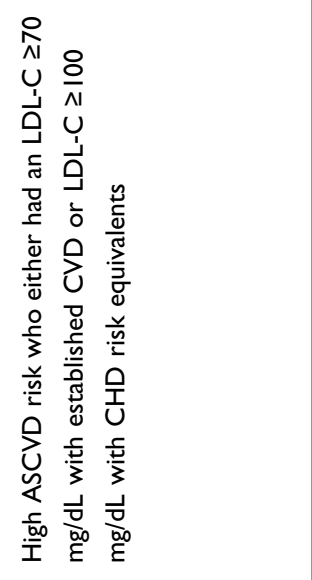 & 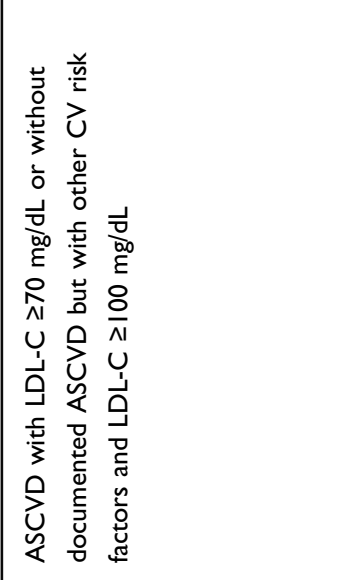 & 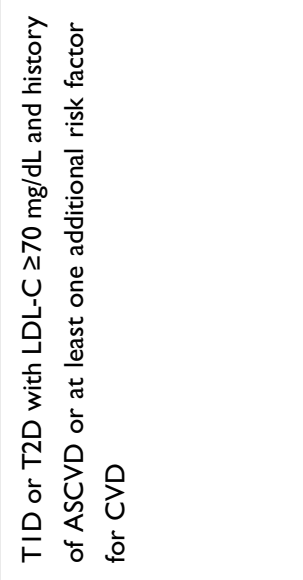 & 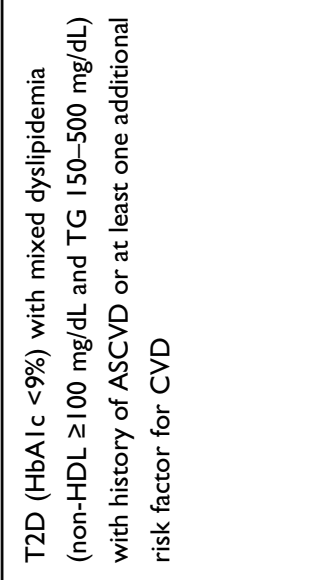 & 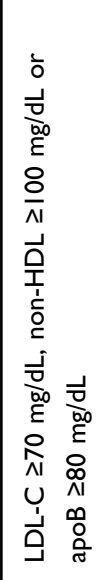 \\
\hline 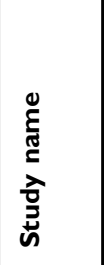 & 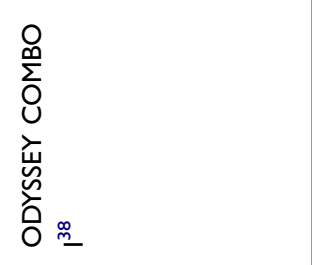 & 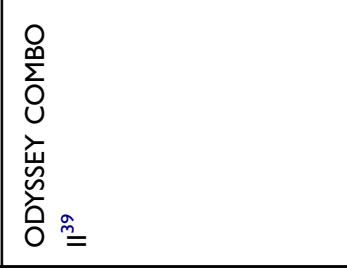 & 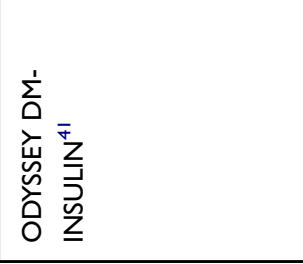 & 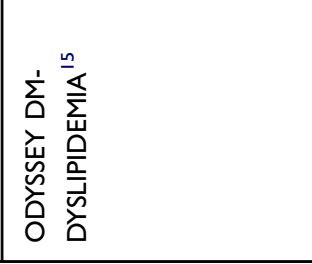 & 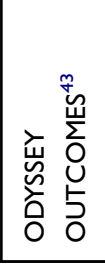 \\
\hline
\end{tabular}




\section{Disclosure}

The authors report no conflicts of interest in this work.

\section{References}

1. Goldstein JL, Brown MS. The low-density lipoprotein pathway and its relation to atherosclerosis. Annu Rev Biochem. 1977;46(1):897930. doi:10.1146/annurev.bi.46.070177.004341

2. Statistics About Diabetes. American Diabetes Association. 2018. Available from: http://www.diabetes.org/diabetes-basics/statistics/. Accessed March 12, 2019.

3. Filippatos TD, Florentin M, Georgoula M, Elisaf MS. Pharmacological management of diabetic. Expert Rev Clin Pharmacol. 2017;10(2):187200. doi:10.1080/17512433.2017.1263565

4. National Diabetes Statistics Report 2017. CDC. 2017. Available from: https://www.cdc.gov/diabetes/pdfs/data/statistics/national-dia betes-statistics-report.pdf. Accessed March 8, 2019.

5. Grundy M, Benjamin IJ, Burke GL, et al. Diabetes and cardiovascular disease: a statement for healthcare professionals from the American Heart Association. Circulation. 1999;100(10):1134-1146. doi:10.1161/01.cir.100.10.1134

6. Feingold KR, Grunfeld C. Diabetes and dyslipidemia. In Endotext [Internet]. MDText. com, Inc. 2018.

7. Garg. Treatment of diabetic dyslipidemia. Am J Cardiol. 1998;81 (4):47B-51B. doi:10.1016/S0002-9149(98)00038-1

8. Handelsman Y, Lepor E. PCSK9 inhibitors in lipid management of patients with diabetes mellitus and high cardiovascular risk. $J \mathrm{Am}$ Heart Assoc. 2018;7(13):e008953. doi:10.1161/JAHA.118.008528

9. Mooradian AD. Dyslipidemia in type 2 diabetes mellitus. Nature. 2009;5(3):150-159.

10. DiPiro T, Talbert L, Yee C, Matzke R, Wells G, Posey. Pharmacotherapy: A Pathophysiologic Approach. 9th ed. New York: McGraw-Hill Education; 2014.

11. Chaudhary R, Garg NS. Sumner. PCSK9 inhibitors: a new era of lipid lowering therapy. World J Cardiol. 2017;9(2):76. doi:10.4330/wjc.v9. i2.76

12. Steinberg D, Witztum L. Inhibition of PCSK9: a powerful weapon for achieving ideal LDL cholesterol levels. Proc Natl Acad Sci. 2009;106 (24):9546-9547. doi:10.1073/pnas.0904560106

13. Feingold, K. R., \& Grunfeld, C. Diabetes and dyslipidemia. In Endotext [Internet]. MDText. com, Inc. 2018.

14. Lagace A. PCSK9 and LDLR degradation: regulatory mechanisms in circulation and in cells. Curr Opin Lipidol. 2014;25(5):987. doi:10.1097/MOL.0000000000000114

15. Ray KK, Leiter LA, Muller-Wieland D, et al. Alirocumab vs usual lipid-lowering care as add-on to statin therapy in individuals with type 2 diabetes and mixed dyslipidaemia: the ODYSSEY DMDYSLIPIDEMIA randomized trial. Diabetes Obes Metab;2018. 1479-1489. doi:10.1111/dom.13257

16. American Diabetes Association. Cardiovascular disease and risk management: standards of medical care in diabetes. 2018. Available from: https://iabetesed.net/wp-content/uploads/2017/12/2018-ADAStandards-of-Care.pdf. Accessed July 31, 2019.

17. Grundy M, Stone NJ, Bailey LA, et al. AHA/ACC/AACVPR/AAPA/ ABC/ACPM/ADA/AGS/APhA/ASPC/NLA/PCNA guideline on the management of blood cholesterol: executive summary. $\mathrm{J} \mathrm{Am}$ Coll Cardiol. 2018. doi:10.1016/j.jacc.2018.11.002

18. Everett BM, Smith RJ, Hiatt WR. Reducing LDL with PCSK9 inhibitors - the clinical benefit of lipid drugs. $N$ Engl $J$ Med. 2015;373(17):1588-1591. doi:10.1056/NEJMp1508120

19. Tavori H, Melone R. Alirocumab: PCSK9 inhibitor for LDL cholesterol reduction. Expert Rev Cardiovasc Ther. 2014;12(10):11371144. doi:10.1586/14779072.2014.954551
20. Zhang J, Tecson M, Rocha A, McCullough A. Usefulness of alirocumab and evolocumab for the treatment of patients with diabetic dyslipidemia. Baylor Univ Med Center Proc. 2018;31(2):180-184. doi:10.1080/08998280.2018.1441255

21. Robinson G, Rosenson S, Farnier, et al. Safety of very low lowdensity lipoprotein cholesterol levels with alirocumab: pooled data from randomized trials. J Am Coll Cardiol. 2017;69(5):417-482. doi:10.1016/j.jacc.2016.11.037

22. Robinson G, Farnier KM,Krempf M, et al. Efficacy and safety of alirocumab in reducing lipids and cardiovascular events. $N$ Engl $J$ Med. 2015;372(16):1489-1499. doi:10.1056/NEJMoa1501031

23. Dadu T, Ballantyne M. Lipid lowering with PCSK9 inhibitors. Nat Rev Cardiol. 2014;11(10):563. doi:10.1038/nrcardio.2014.84

24. Stein EA, Mellis S, Yancopoulos GD, et al. Effect of a monoclonal antibody to PCSK9 on LDL Cholesterol. N Engl J Med;2012. 11081118. doi:10.1056/NEJMoa1 105803

25. Ascending dose study of the safety and tolerability of REGN727 (SAR236553) in healthy volunteers. Clinicaltrials.gov. Available from: https://clinicaltrials.gov/ct2/show/NCT01026597. Accessed August 22, 2019.

26. Ascending dose study of the safety and tolerability of REGN727 (SAR236553) in healthy volunteers. Clinicaltrials.gov. Available from: https://clinicaltrials.gov/ct2/show/NCT01074372. Accessed August 22, 2019.

27. Ascending multi-dose study of REGN727(SAR236553) with and without concomitant atorvastatin. Clinicaltrials.gov. Available from: https:// clinicaltrials.gov/ct2/show/NCT01161082. Accessed August 22, 2019.

28. Stein EA, Gipe D, Bergeron J, et al. Effect of a monoclonal antibody to PCSK9, REGN727/SAR236553, to reduce low-density lopoprotein cholesterol in patients with heterozygois familial hypercholesterolaemia on stable statin dose with or without ezetimibe therapy. Lancet. 2012;380:29-36. doi:10.1016/S0140-6736(12)60771-5

29. Roth EM, McKenney JM, Hanotin C, Asset G, Stein EA. Atorvastatin with or without an antibody to PCSK9 in primary hypercholesterolemia. N Engl J Med. 2012;367:1891-1900. doi:10.10 56/NEJMoa1201832

30. ClinicalTrials.gov. Available from: https://clinicaltrials.gov/ct2/show/ study/NCT01288469. Accessed August 22, 2019.

31. McKenney JM, Koren MJ, Kereiakes DJ, Hanotin C, Ferrand AC, Stein EA. Safety and efficacy of a monoclonal antibody to proprotein convertase subtilisin/kexin type 9 serine protease, SAR236553/ REGN727, in patients with primary hypercholesterolemia receiving ongoing stable atorvastatin therapy. $J \mathrm{Am}$ Coll Cardiol. 2012;59:2344-2353. doi:10.1016/j.jacc.2012.03.007

32. Gaudet D, Kereiakes DJ, McKenney JM, et al. Effect of alirocumab, a monoclonal proprotein convertase subtilisin/kexin 9 antibody, on lipoprotein(a) concentrations (a pooled analysis of $150 \mathrm{mg}$ every two weeks dosing from phase 2 trials). Am J Cardiol. 2014;114:711-715. doi:10.1016/j.amjcard.2014.05.060

33. Kastelein JJP, Ginsberg HN, Langslet G, et al. ODYSSEY FHI and FHII: 78 week results with alirocumab treatment in 735 patients with heterozygous familial hypercholesterolaemia. Eur Heart J. 2015;36:2996-3003.

34. Moriarty PM, Parhofer KG, Babirak SP, et al. Alirocumab in patients with heterozygous familial hypercholesterolaemia undergoing lipoprotein apheresis: the ODYSSEY ESCAPE trial. Eur Heart $J$. 2016;37:3588-3595. doi:10.1093/eurheartj/ehw388

35. Ginsberg HN, Rader DJ, Raat JF, et al. Efficacy and safety of alirocumab in patients with heterozygous familial hypercholesterolemia and LDL-C of $160 \mathrm{mg} / \mathrm{dl}$ or higher. Cardiovasc Drug Ther. 2016;30:473-483. doi:10.1007/s10557-016-6685-y

36. Farnier M, Hovingh GK, Langslet G, et al. Long-term safety and efficacy of alirocumab in patients with heterozygois familial hypercholesterolemia: an open-label extension of the ODYSSEY program. Atherosclerosis. 2018;278:307-314. doi:10.1016/j.atherosclerosis.2018.08.036 
37. ClinicalTrials.gov. Available from: https://clinicaltrials.gov/ct2/show/ NCT01954394. Accessed August 22, 2019.

38. Kereiakes DJ, Robinson JG, Cannon CP, et al. Efficacy and safety of the proprotein convertase subtilisin/kexin type 9 inhibitor alirocumab among high cardiovascular risk patients on maximally tolerated statin therapy: the ODYSSEY COMBO I study. Am Heart J. 2015;169:906-915. doi:10.1016/j.ahj.2015.03.004

39. Cannon CP, Cariou B, Blom D, et al. Efficacy and safety of alirocumab in hihg cardiovascular risk patients with inadequately controlled hypercholesterolaemia on maximally tolerated doses of statins: the ODYSSEY COMBO II randomized controlled trial. Eur Heart J. 2015;36:1186-1194. doi:10.1093/eurheartj/ehv028

40. Leiter LA, Zamorano JL, Bujas-Bobanovic M, et al. Lipid-lowering efficacy and safety of alirocumab in patients with or without diabetes: a sub-analysis of ODYSSEY COMBO II. Diabetes Obes Metab. 2017;19:989-996. doi:10.1111/dom.12909

41. Leiter LA, Cariou B, Muller-Wieland D, et al. Efficacy and safety of alirocumab in insulin-treated individuals with type 1 or type 2 diabetes and high cardiovascular risk: the ODYSSEY DM-INSULIN randomized trial. Diabetes Obes Metab;2017. 1781-1792. doi:10.1111/dom.13114
42. Schwartz GG, Bessac L, Berdan LG, et al. Effect of alirocumab, a monoclonal antibody to PCSK9, on long-term cardiovascular outcomes following acute coronary syndromes: rationale and design of the ODYSSEY outcomes trial. Am Heart $J$. 2014;168:683-689.

43. Schwartz GG, Szarek SM, Bhatt DL, et al. Alirocumab and cardiovascular outcomes after acute coronary syndrome. $N$ Engl $\mathrm{J}$ Med. 2018;379(22):2097-2107.

44. Ray KK, Colhoun HM, Szarek M, et al. Alirocumab and Cardiovascular OUTCOMES in Patients with Acute Coronary Syndrome (ACS) and Diabetes: Prespecified Analyses of ODYSSEY OUTCOMES. American Diabetes Association; 2018.

45. Colhoun HM, Ginsberg HN, Robinson JG, et al. No effect of PCSK9 inhibitor alirocumab on the incidence of diabetes in a pooled analysis from 10 ODYSSEY Phase 3 studies. Eur Heart J. 2016;37:29812989. doi:10.1093/eurheartj/ehw292

46. Leiter LA, Tinahones FJ, Karalis DG, et al. Alirocumab safety in people with and without diabetes mellitus: pooled data from 14 ODYSSEY trials. Diabet Med;2018. 1742-1751. doi:10.1111/ dme. 13817

Diabetes, Metabolic Syndrome and Obesity: Targets and Therapy

Dovepress

\section{Publish your work in this journal}

Diabetes, Metabolic Syndrome and Obesity: Targets and Therapy is an international, peer-reviewed open-access journal committed to the rapid publication of the latest laboratory and clinical findings in the fields of diabetes, metabolic syndrome and obesity research. Original research, review, case reports, hypothesis formation, expert opinion and commentaries are all considered for publication. The manuscript management system is completely online and includes a very quick and fair peer-review system, which is all easy to use. Visit http://www.dovepress.com/testimonials.php to read real quotes from published authors. 